Article

\title{
Picard-Jungck Operator for a Pair of Mappings and Simulation Type Functions
}

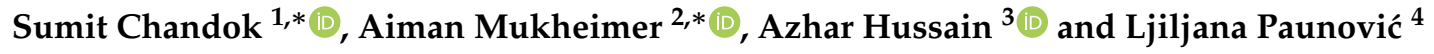 \\ School of Mathematics, Thapar Institute of Engineering and Technology, Patiala 147004, India \\ 2 Department of Mathematics and General Sciences, Prince Sultan University, Riyadh 11586, Saudi Arabia \\ 3 Department of Mathematics, University of Sargodha, Sargodha 40100, Pakistan; hafiziqbal30@yahoo.com \\ 4 Teacher Education School in Prizren-Leposavić, University of Priština, Leposavić 43500, Serbia; \\ lijijana.paunovic76@gmail.com \\ * Correspondence: sumit.chandok@thapar.edu (S.C.); mukheimer@psu.edu.sa (A.M.)
}

Received: 24 April 2019; Accepted: 18 May 2019; Published: 22 May 2019

\begin{abstract}
In this manuscript, we propose a new class of Picard-Jungck operators for a pair of mappings on complete metric spaces by taking into account of the $\mathscr{C}_{\mathscr{G}}$-simulation function. Also, some new results for the existence of such operators for a pair of self mappings in the setting of metric spaces are obtained. Some nontrivial examples are presented to show the usability of the results.
\end{abstract}

Keywords: point of coincidence; Picard-Jungck operators; weakly compatible mappings; $\mathscr{C}_{\mathscr{g}}$-simulation function

MSC: 55M20; 47H10

\section{Introduction}

The study of the Picard operators is similar to the study of contractive-type mappings in the context of metric spaces. It is easy to see that almost all contractive-type mappings on a complete metric space are Picard operators. In the present paper, we propose a new class of Picard-Jungck operators for a pair of mappings on complete metric spaces by taking into account of the $\mathscr{C}_{g}$-simulation function. Also some new results for the existence of such operators for a pair of self mappings in the setting of metric spaces are obtained. Some nontrivial examples are also given to show the usability of the results. The result proved here are short and generalize many known results from the literature. For different variants of simulation function we can obtain very interesting results.

To begin with, we have the following notations, definitions and results which will be used in the sequel.

Definition 1. (See $[1,2]$ ) We define $\mathscr{C}$-class function as a family of continuous mappings $\mathscr{G}:[0,+\infty)^{2} \rightarrow \mathbb{R}$ and satisfies the following conditions:

(1) $\mathscr{G}(u, v) \leq u$;

(2) $\mathscr{G}(u, v)=u \Leftrightarrow$ either $u=0$ or $v=0$, for all $u, v \in[0,+\infty)$.

Employing a family of $\mathscr{C}$-class function, authors [1,2] generalized the class of simulation functions introduced by Khojasteh et al. ([3]) as follows:

Definition 2. A mapping $\mathscr{G}:[0,+\infty)^{2} \rightarrow \mathbb{R}$ has the property $\mathscr{C}_{\mathscr{G}}$, if there exists an $\mathscr{C}_{\mathscr{G}} \geq 0$ such that

(1) $\mathscr{G}(u, v)>\mathscr{C}_{\mathscr{G}}$ implies $u>v$;

(2) $\mathscr{G}(v, v) \leq \mathscr{C}_{g}$, for all $v \in[0,+\infty)$.

For examples of $\mathscr{C}$-class functions that have property $\mathscr{C}_{\mathscr{G}}$ see $[1,2,4]$. 
Definition 3. (see [1]) We define $\mathscr{Z}_{\mathscr{G}}$ as the family of all $\mathscr{G}_{\mathscr{G}}$-simulation functions $\zeta:[0,+\infty)^{2} \rightarrow \mathbb{R}$ satisfying the following:

(1) $\zeta(v, u)<\mathscr{G}(u, v)$ for all $v, u>0$, where $\mathscr{G}:[0,+\infty)^{2} \rightarrow \mathbb{R}$ is a $\mathscr{C}$-class function;

(2) if $\left\{v_{n}\right\},\left\{u_{n}\right\}$ are sequences in $(0,+\infty)$ such that $\lim _{n \rightarrow \infty} v_{n}=\lim _{n \rightarrow \infty} u_{n}>0$, and $v_{n}<u_{n}$, then $\limsup _{n \rightarrow \infty} \zeta\left(v_{n}, u_{n}\right)<\mathscr{C}_{\mathscr{G}}$.

Each simulation function as in article [3] is also a $\mathscr{C}_{\mathscr{G}}$-simulation function as defined in Definition 3, but the converse of this is not true. For this see Example 3.3 of [5] using the $\mathscr{C}$-class function $\mathscr{G}(u, v)=$ $u-v$. For examples of simulation functions and $\mathscr{C}_{\mathscr{G}}$-simulation functions see [1-12].

Let $f$ and $g$ act naturally maps on a set $\mathscr{X}$. Review that if $w=f \omega=g \omega$ for some $\omega \in \mathscr{X}$, at that point $\omega$ is known as a coincidence point of $f$ and $g$, and $w$ is known as a point of coincidence of $f$ and $g$. The pair $(f, g)$ is weakly compatible if $f$ and $g$ commute at their coincidence points.

The following result will be needed in the sequel.

Lemma 1. (see $[2,13])$ Let $\left\{\omega_{n}\right\}$ be a sequence in a metric space $(\mathscr{X}, d)$ such that

$$
\lim _{n \rightarrow \infty} d\left(\omega_{n}, \omega_{n+1}\right)=0
$$

If $\left\{\omega_{n}\right\}$ is not a Cauchy in $\mathscr{X}$, then there exist $\varepsilon>0$ and two sequences $\left\{\omega_{m(k)}\right\}$ and $\left\{\omega_{n(k)}\right\}$ of positive integers such that $n(k)>m(k)>k$ and the following sequences tend to $\varepsilon^{+}$when $k \rightarrow+\infty$ :

$$
\begin{gathered}
d\left(\omega_{m(k)}, \omega_{n(k)}\right), d\left(\omega_{m(k)}, \omega_{n(k)+1}\right), d\left(\omega_{m(k)-1}, \omega_{n(k)}\right), \\
d\left(\omega_{m(k)-1}, \omega_{n(k)+1}\right), d\left(\omega_{m(k)+1}, \omega_{n(k)+1}\right) .
\end{gathered}
$$

\section{Results}

In this section, we institute some results on the existence of Picard-Jungck operator (PJO) for a pair of mappings by using simulation functions in the structure of metric spaces. We begin with the following:

A sequence $\left\{\omega_{n}\right\}_{n \in \mathbb{N} \cup\{0\}} \subseteq \mathscr{X}$ is a Picard-Jungck sequence (PJS) of the pair $(f, g)$ (based on $\left.\omega_{0}\right)$ if $\xi_{n}=f \omega_{n}=f^{n} \omega_{0}=g \omega_{n+1}=g^{n+1} \omega_{0}$ for all $n \in \mathbb{N} \cup\{0\}$ (see also ([5] Definition 4.4)).

A pair $(f, g)$ is said to be a weakly Picard-Jungck operator (WPJO) if it has a unique point of coincidence point $z \in \mathscr{X}$ and $z=\lim _{n \rightarrow \infty} f^{n} u=g^{n+1} u$ for all $u \in \mathscr{X}$.

A pair $(f, g)$ is said to be a Picard-Jungck operator (PJO) if it has a unique common fixed point $u \in \mathscr{X}$ and $u=\lim _{n \rightarrow \infty} f^{n} u=g^{n+1} u$ for all $u \in \mathscr{X}$.

A self-mapping $f$ is said to be a Picard operator (PO) if it has a unique fixed point $z \in \mathscr{X}$ and $z=\lim _{n \rightarrow \infty} f^{n} u$ for all $u \in \mathscr{X}$.

Definition 4. Let $(f, g)$ be a pair of self mappings on a metric space $(\mathscr{X}, d)$. An operator $f$ is called a $\left(\mathscr{E}_{\mathscr{G}}, g\right)$-contraction if there exists $\zeta \in \mathscr{X}_{\mathscr{G}}$ such that for all $\omega, \xi \in \mathscr{X}$ with $g \omega \neq g \xi$, we have

$$
\zeta(d(f \omega, f \xi), L(\omega, \xi)) \geq \mathscr{C}_{\mathscr{G}},
$$

where

$$
L(\omega, \xi)=\max \{d(g \omega, g \xi), d(g \omega, f \omega), d(g \xi, f \xi)\}
$$

Now to state our first new result for the notion of $\left(\mathscr{E}_{\mathscr{G}}, g\right)$-contraction, we need the following result. 
Lemma 2. Let $(f, g)$ be a pair of self mappings on a metric space $(\mathscr{X}, d)$ and $f$ be a $\left(\mathscr{Z}_{\mathscr{G}}, g\right)$-contraction. Suppose that there exists a PJS $\left\{\xi_{n}\right\}_{n \in \mathbb{N} \cup\{0\}}$ of $(f, g)$. Then the sequence $\left\{d\left(\xi_{n}, \xi_{n+1}\right)\right\}$ is decreasing and $d\left(\xi_{n}, \xi_{n+1}\right) \rightarrow 0$ as $n \rightarrow \infty$.

Proof. Suppose that there is a PJS $\left\{\xi_{n}\right\}$ such that $\xi_{n}=f \omega_{n}=g \omega_{n+1}$ where $n \in \mathbb{N} \cup\{0\}$. Suppose that $\xi_{n} \neq \xi_{n+1}$ for all $n \in \mathbb{N} \cup\{0\}$. Substituting $\omega=\omega_{n+1}, \xi=\omega_{n+2}$ in (1) we obtain that

$$
\mathscr{C}_{\mathscr{G}} \leq \zeta\left(d\left(f \omega_{n+1}, f \omega_{n+2}\right), L\left(\omega_{n+1}, \omega_{n+2}\right)\right)
$$

where

$$
\begin{aligned}
L\left(\omega_{n+1}, \omega_{n+2}\right) & =\max \left\{d\left(g \omega_{n+1}, g \omega_{n+2}\right), d\left(g \omega_{n+1}, f \omega_{n+1}\right), d\left(g \omega_{n+2}, f \omega_{n+2}\right)\right\} \\
& =\max \left\{d\left(\xi_{n+1}, \xi_{n+2}\right), d\left(\xi_{n}, \xi_{n+1}\right)\right\} .
\end{aligned}
$$

If $\max \left\{d\left(\xi_{n+1}, \xi_{n+2}\right), d\left(\xi_{n}, \xi_{n+1}\right)\right\}=d\left(\xi_{n+1}, \xi_{n+2}\right)$, we have

$$
\begin{aligned}
\mathscr{C}_{\mathscr{G}} & \leq \zeta\left(d\left(f \omega_{n+1}, f \omega_{n+2}\right), L\left(\omega_{n+1}, \omega_{n+2}\right)\right)=\zeta\left(d\left(\xi_{n+1}, \xi_{n+2}\right), d\left(\xi_{n+1}, \xi_{n+2}\right)\right) \\
& <\mathscr{G}\left(d\left(\xi_{n+1}, \xi_{n+2}\right), d\left(\xi_{n+1}, \xi_{n+2}\right)\right)
\end{aligned}
$$

which is a contradiction using (2) of Definition 2.

Therefore, $\max \left\{d\left(\xi_{n+1}, \xi_{n+2}\right), d\left(\xi_{n}, \xi_{n+1}\right)\right\}=d\left(\xi_{n}, \xi_{n+1}\right)$. Hence we have

$$
\begin{aligned}
\mathscr{C}_{\mathscr{G}} & \leq \zeta\left(d\left(f \omega_{n+1}, f \omega_{n+2}\right), L\left(\omega_{n+1}, \omega_{n+2}\right)\right)=\zeta\left(d\left(\xi_{n+1}, \xi_{n+2}\right), d\left(\xi_{n}, \xi_{n+1}\right)\right) \\
& <\mathscr{G}\left(d\left(\xi_{n}, \xi_{n+1}\right), d\left(\xi_{n+1}, \xi_{n+2}\right)\right) .
\end{aligned}
$$

Using (1) of Definition 2, we have $d\left(\xi_{n}, \xi_{n+1}\right)>d\left(\xi_{n+1}, \xi_{n+2}\right)$. Hence, for all $n \in \mathbb{N} \cup\{0\}$ we get that $d\left(\xi_{n+1}, \xi_{n+2}\right)<d\left(\xi_{n}, \xi_{n+1}\right)$.

Further we have to prove that $\xi_{n} \neq y_{m}$ for $n \neq m$. Indeed, suppose that $\xi_{n}=y_{m}$ for some $n>m$. So we choose $\omega_{n+1}=\omega_{m+1}$ (which is obviously possible by the definition of PJS $\left\{\xi_{n}\right\}$ ) and hence also $\xi_{n+1}=\xi_{m+1}$. Then following the previous arguments, we have

$$
d\left(\xi_{n}, \xi_{n+1}\right)<d\left(\xi_{n-1}, \xi_{n}\right)<\cdots<d\left(y_{m}, \xi_{m+1}\right)=d\left(\xi_{n}, \xi_{n+1}\right)
$$

which is a contradiction.

Therefore there exists $D \geq 0$ such that $\lim _{n \rightarrow \infty} d\left(\xi_{n}, \xi_{n+1}\right)=D \geq 0$. Suppose that $D>0$. Since $d\left(\xi_{n+1}, \xi_{n+2}\right)<d\left(\xi_{n}, \xi_{n+1}\right)$ and both $d\left(\xi_{n+1}, \xi_{n+2}\right)$ and $d\left(\xi_{n}, \xi_{n+1}\right)$ tend to $D$, using (2) of Definition 3, we get

$$
\mathscr{C}_{\mathscr{G}} \leq \limsup _{n \rightarrow \infty} \zeta\left(d\left(\xi_{n+1}, \xi_{n+2}\right), d\left(\xi_{n}, \xi_{n+1}\right)\right)<\mathscr{C}_{\mathscr{G}}
$$

which is a contradiction. Hence $\lim _{n \rightarrow \infty} d\left(\xi_{n}, \xi_{n+1}\right)=D=0$.

Lemma 3. Let $(f, g)$ be a pair of self mappings on a metric space $(\mathscr{X}, d)$ and $f$ be a $\left(\mathscr{Z}_{\mathscr{G}}, g\right)$-contraction. Suppose that there exists a PJS $\left\{\xi_{n}\right\}_{n \in \mathbb{N} \cup\{0\}}$ of $(f, g)$. Then the PJS $\left\{\xi_{n}\right\}$ is a Cauchy sequence.

Proof. Suppose that there is a PJS $\left\{\xi_{n}\right\}$ such that $\xi_{n}=f \omega_{n}=g \omega_{n+1}$ where $n \in \mathbb{N} \cup\{0\}$.

If $\xi_{k}=\xi_{k+1}$ for some $k \in \mathbb{N} \cup\{0\}$, then $g \omega_{k+1}=\xi_{k}=\xi_{k+1}=f \omega_{k+1}$ and $f$ and $g$ have a point of coincidence. Therefore, suppose that $\xi_{n} \neq \xi_{n+1}$ for all $n \in \mathbb{N} \cup\{0\}$. Using Lemma 2, we have $d\left(\xi_{n+1}, \xi_{n+2}\right)<d\left(\xi_{n}, \xi_{n+1}\right)$ for all $n \in \mathbb{N} \cup\{0\}$ and $\lim _{n \rightarrow \infty} d\left(\xi_{n}, \xi_{n+1}\right)=0$. 
Now, we have to show that $\left\{\xi_{n}\right\}$ is a Cauchy sequence. Suppose, to the contrary, that it is not. Putting $\omega=\omega_{m(k)+1}, \xi=\omega_{n(k)+1}$ in (1), we obtain

$$
\begin{aligned}
\mathscr{C}_{\mathscr{G}} & \leq \zeta\left(d\left(f \omega_{m(k)+1}, f \omega_{n(k)+1}\right), L\left(\omega_{m(k)+1}, \omega_{n(k)+1}\right)\right) \\
& <G\left(L\left(\omega_{m(k)+1}, \omega_{n(k)+1}\right), d\left(\xi_{m(k)+1}, \xi_{n(k)+1}\right)\right),
\end{aligned}
$$

where

$$
\begin{aligned}
L\left(\omega_{m(k)+1}, \omega_{n(k)+1}\right) & =\max \left\{d\left(g \omega_{m(k)+1}, g \omega_{n(k)+1}\right), d\left(g \omega_{m(k)+1}, f \omega_{m(k)+1}\right),\right. \\
d & \left.\left(g \omega_{n(k)+1}, f \omega_{n(k)+1}\right)\right\} \\
& =\max \left\{d\left(\xi_{m(k)}, \xi_{n(k)}\right), d\left(\xi_{m(k)}, \xi_{m(k)+1}\right), d\left(\xi_{n(k)}, \xi_{n(k)+1}\right)\right\} .
\end{aligned}
$$

Now, since the sequence $\left\{\xi_{n}\right\}$ is not a Cauchy sequence, by Lemma 1 , we have $d\left(\xi_{m(k)}, \xi_{n(k)}\right)$, $d\left(\xi_{m(k)+1}, \xi_{n(k)+1}\right), d\left(\xi_{m(k)}, \xi_{n(k)+1}\right)$ and $d\left(\xi_{n(k)}, \xi_{m(k)+1}\right)$ tend to $\varepsilon>0$, as $k \rightarrow \infty$. Therefore, using (2), we have

$$
\mathscr{C}_{\mathscr{G}} \leq \limsup _{n \rightarrow \infty} \zeta\left(d\left(\xi_{m(k)+1}, \xi_{n(k)+1}\right), L\left(\omega_{m(k)+1}, \omega_{n(k)+1}\right)\right)<\mathscr{C}_{\mathscr{G}}
$$

which is a contradiction. Therefore, the PJS $\left\{\xi_{n}\right\}$ is a Cauchy sequence.

Now, we recall the following result of Abbas and Jungck [14] to be used in the sequel.

Proposition 1. Let $f$ and $g$ be weakly compatible self maps of a set $\mathscr{X}$. If $f$ and $g$ have a unique point of coincidence $w=f \omega=g \omega$, then $w$ is a unique common fixed point of $f$ and $g$.

Theorem 1. Let $(f, g)$ be a pair of self mappings on a metric space $(\mathscr{X}, d)$ and $f$ be a $\left(\mathscr{Z}_{\mathscr{G}}, g\right)$-contraction. Suppose that there exists a PJS $\left\{\xi_{n}\right\}_{n \in \mathbb{N} \cup\{0\}}$ of $(f, g)$. Also assume that at least one of the following conditions hold:

(i) $(f(\mathscr{X}), d)$ or $(g(\mathscr{X}), d)$ is complete;

(ii) $(\mathscr{X}, d)$ is complete, $g$ is continuous and $g(x)$ is closed subspace of $\mathscr{X}$.

Then pair $(f, g)$ is WPJO. Moreover, if $f$ and $g$ are weakly compatible, then pair $(f, g)$ is PJO.

Proof. First of all we shall prove that the point of coincidence of $f$ and $g$ is unique (if it exists). Suppose that $z_{1}$ and $z_{2}$ are distinct points of coincidence of $f$ and $g$. From this it follows that there exist two points $v_{1}$ and $v_{2}\left(v_{1} \neq v_{2}\right)$ such that $f v_{1}=g v_{1}=z_{1}$ and $f v_{2}=g v_{2}=z_{2}$. Then (1) implies that

$$
\mathscr{C}_{\mathscr{G}} \leq \zeta\left(d\left(f v_{1}, f v_{2}\right), L\left(v_{1}, v_{2}\right)\right)
$$

where

$$
L\left(v_{1}, v_{2}\right)=\max \left\{d\left(g v_{1}, g v_{2}\right), d\left(g v_{1}, f v_{1}\right), d\left(g v_{2}, f v_{2}\right)\right\}=d\left(z_{1}, z_{2}\right) .
$$

Therefore, we have

$$
\mathscr{C}_{\mathscr{G}} \leq \zeta\left(d\left(f v_{1}, f v_{2}\right), L\left(v_{1}, v_{2}\right)\right)=\zeta\left(d\left(z_{1}, z_{2}\right), d\left(z_{1}, z_{2}\right)\right)<G\left(d\left(z_{1}, z_{2}\right), d\left(z_{1}, z_{2}\right)\right) \leq \mathscr{C}_{\mathscr{G}},
$$

which is a contradiction.

In order to prove that a pair $(f, g)$ is WPJO, suppose that there is a PJS $\left\{\xi_{n}\right\}$ such that $\xi_{n}=f \omega_{n}=$ $g \omega_{n+1}$ where $n \in \mathbb{N} \cup\{0\}$.

If $\xi_{k}=\xi_{k+1}$ for some $k \in \mathbb{N} \cup\{0\}$, then $g \omega_{k+1}=\xi_{k}=\xi_{k+1}=f \omega_{k+1}$ and $f$ and $g$ have a point of coincidence. Therefore, suppose that $\xi_{n} \neq \xi_{n+1}$ for all $n \in \mathbb{N} \cup\{0\}$. Using Lemma 2, we have 
$d\left(\xi_{n+1}, \xi_{n+2}\right)<d\left(\xi_{n}, \xi_{n+1}\right)$ for all $n \in \mathbb{N} \cup\{0\}$ and $\lim _{n \rightarrow \infty} d\left(\xi_{n}, \xi_{n+1}\right)=0$. Now, using Lemma 3, we obtain that PJS $\left\{\xi_{n}\right\}$ is a Cauchy sequence.

Suppose that (i) holds, i.e., $(g(X), d)$ is complete. Then there exists $v \in \mathscr{X}$ such that $\xi_{n-1}=$ $f \omega_{n-1}=g \omega_{n} \rightarrow g v$ as $n \rightarrow \infty$. We shall prove that $f v=g v$. It is clear that we can suppose $\xi_{n} \neq f v, g v$ for all $n \in \mathbb{N} \cup\{0\}$. Suppose that $d(f v, g v)>0$ and using (1), we have

$$
\mathscr{C}_{\mathscr{G}} \leq \zeta\left(d\left(f \omega_{n}, f v\right), L\left(\omega_{n}, v\right)\right)<G\left(L\left(\omega_{n}, v\right), d\left(f \omega_{n}, f v\right)\right),
$$

where

$$
L\left(\omega_{n}, v\right)=\max \left\{d\left(g \omega_{n}, g v\right), d\left(g \omega_{n}, f \omega_{n}\right), d(g v, f v)\right\} .
$$

Taking $n \rightarrow \infty$, we have

$$
\mathscr{C}_{\mathscr{G}} \leq \zeta(d(g v, f v), d(g v, f v))<G(d(g v, f v), d(g v, f v)) .
$$

Using (2) of Definition 2, we get

$$
\mathscr{C}_{\mathscr{G}} \leq \zeta(d(g v, f v), d(g v, f v))<G(d(g v, f v), d(g v, f v)) \leq \mathscr{C}_{\mathscr{G}},
$$

which is a contradiction. Hence, $f v=g v$ is a (unique) point of coincidence of $f$ and $g$.

Similarly, we can prove that $f v=g v$ is a (unique) point of coincidence of $f$ and $g$, when $(f(\mathscr{X}), d)$ is complete.

Finally, suppose that (ii) holds. Since $(\mathscr{X}, d)$ is complete, then there exists $v \in \mathscr{X}$ such that $\xi_{n}=f \omega_{n} \rightarrow v$, when $n \rightarrow \infty$. As $g$ is continuous, and $g(\mathscr{X})$ is a closed subspace of $\mathscr{X}$. Then, we choose $u \in \mathscr{X}$ such that $\xi_{n}=g\left(\omega_{n+1}\right) \rightarrow g(u)=v$ when $n \rightarrow \infty$. Suppose that $d(f u, v)>0$. Consider

$$
\mathscr{C}_{\mathscr{G}} \leq \zeta\left(d\left(f\left(\omega_{n}\right), f u\right), L\left(\omega_{n}, u\right)\right)<G\left(L\left(\omega_{n}, u\right), d\left(f\left(\varpi_{n}\right), f u\right)\right),
$$

where $L\left(\omega_{n}, u\right)=\max \left\{d\left(g\left(\omega_{n}\right), g u\right), d\left(g\left(\omega_{n}\right), f\left(\omega_{n}\right)\right), d(g u, f u)\right\}$.

Taking $n \rightarrow \infty$, we have $\mathscr{C}_{G} \leq \zeta\left(d(v, f u), L\left(\omega_{n}, u\right)\right)<G\left(L\left(\omega_{n}, u\right), d\left(f\left(\omega_{n}\right), f u\right)\right)=$ $G(d(v, f u), d(v, f u))$. Using (2) of Definition 2, we get $\mathscr{C}_{\mathscr{G}} \leq \zeta(d(v, f u), d(v, f u))<$ $G(d(v, f u), d(v, f u)) \leq \mathscr{C}_{g}$, which is a contradiction. Hence, $f u=g u=v$ is a (unique) point of coincidence of $f$ and $g$. Hence, the result is proved in both cases.

Further, since the pair $(f, g)$ is weakly compatible, then according to Proposition 1, they have a unique common fixed point.

Corollary 1. Let $(f, g)$ be a pair of self mappings on a metric space $(X, d)$ and $f$ satisfies

$$
\zeta(d(f \omega, f \xi), d(g \omega, g \xi)) \geq \mathscr{C}_{\mathscr{G}},
$$

for all $\omega, \xi \in \mathscr{X}$ with $g \omega \neq g \xi$ and $\zeta \in \mathscr{X}_{\mathscr{G}}$. Suppose that there exists a PJS $\left\{\xi_{n}\right\}_{n \in \mathbb{N} \cup\{0\}}$ of $(f, g)$. Also assume that at least one of the following conditions hold:

(i) $(f(X), d)$ or $(g(X), d)$ is complete;

(ii) $(\mathscr{X}, d)$ is complete, $g$ is continuous and $g(\mathscr{X})$ is closed subspace of $\mathscr{X}$.

Then pair $(f, g)$ is WPJO. Moreover, if $f$ and $g$ are weakly compatible, then pair $(f, g)$ is PJO.

Corollary 2. Let $f$ be a self mapping on a complete metric space $(\mathscr{X}, d)$ and $f$ satisfies

$$
\zeta(d(f \omega, f \xi), d(\omega, \xi)) \geq \mathscr{C}_{\mathscr{G}},
$$

for all $\omega, \xi \in \mathscr{X}$ with $\omega \neq \xi$ and $\zeta \in \mathscr{X} \mathscr{G}$. Suppose that there exists a Picard sequence $\left\{u_{n}\right\} \subseteq \mathscr{X}$ defined by $u_{n+1}=f^{n} u_{0}=f u_{n}$ for all $n \in \mathbb{N} \cup\{0\}$. Then $f$ is a PO. 


\section{Discussion}

Theorem 1 and Corollary 1 hold true if, in particular, $(\mathscr{X}, d)$ is complete, $g$ is continuous and $f$ and $g$ are commuting.

It is also worth noting that the two examples given in [5] are not suitable to support their main result. Neither of these examples is a proper generalization of the corresponding result of Jungck [15]. In other words, there is a $\lambda \in(0,1)$ such that $d(f \omega, f \xi) \leq \lambda d(g \omega, g \xi)$ for all $\omega, \xi \in \mathscr{X}=$ $[0,+\infty), f \omega=\omega+10, g \omega=\frac{10}{9} \omega+e^{\omega}+\sin \frac{\pi \omega}{2(1+\omega)}+1, d(\omega, \xi)=|\omega-\xi|,(([5]$ Example 5.11)), resp. $f \omega=\arctan (\omega+2), g \omega=\log (\omega+3)(([5]$ Example 5.12)). Also, ([4] Example 20), in the same metric space, where $f \omega=\omega+2, g \omega=4 \omega+e^{2 \omega}$ is such.

The following example guarantees the existence and uniqueness of a solution for a nonlinear equation.

Example 1. Let $\mathscr{X}=[0,+\infty)$ be endowed with the usual metric $d(\omega, \xi)=|\omega-\xi|$ for all $\omega, \xi \in[0,+\infty)$, and consider the mappings $f, g:[0,+\infty) \rightarrow[0,+\infty)$ given, for all $\omega \in[0,+\infty)$, by

$$
f \omega=\omega+2, \quad g \omega=4 \omega+e^{2 \omega} .
$$

Assume that we have to solve the following nonlinear equation

$$
\omega+2=4 \omega+e^{2 \omega}
$$

Corollary 1 can be applied using the simulation function $\zeta(v, u)=\frac{9}{10}\left(u-\frac{(2+v) v}{1+v}\right)$ for $u, v \in[0,+\infty)$ and $\mathscr{C}_{\mathscr{F}}=0, \mathscr{F}(u, v)=u-\frac{(2+v) v}{1+v}$. Now, we have that

$$
\begin{aligned}
\zeta(d(f \omega, f \xi), d(g \omega, g \xi)) & =\frac{9}{10}\left(d(g \omega, g \xi)-\frac{(2+d(f \omega, f \xi)) d(f \omega, f \xi)}{1+d(f \omega, f \xi)}\right) \\
& =\frac{9}{10}\left(\left|4(\omega-\xi)+\left(e^{2 \omega}-e^{2 \xi}\right)\right|-\frac{(2+|\omega-\xi|)|\omega-\xi|}{1+|\omega-\xi|}\right) \\
& \geq 0 .
\end{aligned}
$$

Since $f(X)=[2,+\infty), g(\mathscr{X})=[1,+\infty)$, using Corollary $1(i)$ the result follows.

The following example shows that our Theorem 1 is a proper generalization of the corresponding results of Jungck [15], L.-de-Hierro et al. [5] and of Olgun et al. [16].

Example 2. Let $\mathscr{X}=[0,1]$ and $d: \mathscr{X} \times \mathscr{X} \rightarrow[0,+\infty)$ be defined by $d(\omega, \xi)=|\omega-\xi|$. Then $(\mathscr{X}, d)$ is a complete metric space. Define $f, g: X \rightarrow X$ as $f \omega=\frac{\omega}{2+\omega}, g \omega=\frac{\mathscr{W}}{2}$. Then, $f$ is not Jungck's contraction in the sense that there is $\lambda \in(0,1)$ such that $d(f \omega, f \xi) \leq \lambda d(g \omega, g \xi)$ for all $\omega, \xi \in \mathscr{X}$. However, putting $\zeta(v, u)=\frac{u}{u+1}-v, \mathscr{G}(u, v)=u-v, \mathscr{C}_{\mathscr{G}}=0$, we have that $f$ is $a(\mathcal{Z}, g)$-contraction with respect to $\zeta$. Indeed, we obtain

$$
\begin{aligned}
\zeta(d(f \omega, f \xi), d(g \omega, g \xi)) \geq \mathscr{C}_{\mathscr{G}}=0 \text { if and only if } \frac{d(g \omega, g \xi)}{1+d(g \omega, g \xi)}-d(f \omega, f \xi) \geq 0 \\
\Leftrightarrow \frac{\frac{1}{2}|\omega-\xi|}{1+\frac{1}{2}|\omega-\xi|}-\left|\frac{x}{x+2}-\frac{\xi}{\xi+2}\right| \\
\quad=\frac{|\omega-\xi|}{2+|\omega-\xi|}-\frac{2|\omega-\xi|}{(\omega+2)(\xi+2)} \geq 0,
\end{aligned}
$$


whenever $\omega, \xi \in \mathscr{X}$. Further, since $f(\mathscr{X})=\left[0, \frac{1}{3}\right] \subseteq\left[0, \frac{1}{2}\right]=g(\mathscr{X})$ there exists a Picard-Jungck sequence $\left\{\omega_{n}\right\}_{n \in \mathbb{N} \cup\{0\}}$ of $(f, g)$. As both $(f(\mathscr{X}), d)$ or $(g(\mathscr{X}), d)$ are complete, this means that all the conditions of Corollary 1 are satisfied, i.e., the mappings $f$ and $g$ have a coincidence point $\omega=0$. In other words, they have a unique common fixed point, which is the only solution of equation $\frac{\omega}{\omega+2}=\frac{\omega}{2}, \omega \in[0,1]$.

Author Contributions: Conceptualization, S.C. and A.M.; formal analysis, S.C., A.H. and L.P.; investigation, L.P.; writing-original draft preparation, S.C.; writing-review and editing, A.H.; supervision, S.C. and A.M.; funding acquisition, A.M.

Funding: The second author would like to thank Prince Sultan University for funding this work through research group Nonlinear Analysis Methods in Applied Mathematics (NAMAM) group number RG-DES-2017-01-17.

Acknowledgments: The authors would like to thanks Professor Stojan Radenović for his support during the preparation of this manuscript.

Conflicts of Interest: The authors declare no conflict of interest.

\section{Abbreviations}

The following abbreviations are used in this manuscript:

PO Picard operator

PJO Picard-Jungck operator

PJS Picard-Jungck sequence

WPJO Weakly Picard-Jungck operator

\section{References}

1. Liu, X.L.; Ansari, A.H.; Chandok, S.; Radenović, S. On some results in metric spaces using auxiliary simulation functions via new functions. J. Comput. Anal. Appl. 2018, 24, 1103-1114.

2. Radenović, S.; Chandok, S. Simulation type functions and coincidence points. Filomat 2018, 32, 141-147. [CrossRef]

3. Khojasteh, F.; Shukla, S.; Radenović, S. A new approach to the study of fixed point theorems via simulation functions. Filomat 2015, 29, 1189-1194. [CrossRef]

4. Ansari, A.H.; Isik, H.; Radenović, S. Coupled fixed point theorems for contractive mappings involving new function classes and applications. Filomat 2017, 31, 1893-1907. [CrossRef]

5. L.-de-Hierro, A.F.R.; Karapınar, E.; R.-L.-de-Hierro, C.; M.-Morenoa, J. Coincidence point theorems on metric spaces via simulation functions. J. Comput. Appl. Math. 2015, 275, 345-355. [CrossRef]

6. Nastasi, A.; Vetro, P. Fixed point results on metric and partial metric spaces via simulation functions. J. Nonlinear Sci. Appl. 2015, 8, 1059-1069. [CrossRef]

7. Wang, S.; Ansari, A.H.; Chandok, S. Some fixed point results for non-decreasing and mixed monotone mappings with auxiliary functions. Fixed Point Theory Appl. 2015. [CrossRef]

8. Abbas, M.; Latif, A.; Suleiman, Y. Fixed points for cyclic R-contractions and solution of nonlinear Volterra integro-differential equations. Fixed Point Theory Appl. 2016. [CrossRef]

9. Chanda, A.; Dey, L.K.; Radenović, S. Simulation functions: A Survey of recent results. RACSAM 2018. [CrossRef]

10. L.de-Hierro, A.F.R.; Shahzad, N. New fixed point theorem under R-contractions. Fixed Point Theory Appl. 2015. [CrossRef]

11. L.de-Hierro, A.F.R.; Shahzad, N. Common fixed point theorems under (R,S)-contractivity conditions. Fixed Point Theory Appl. 2016. [CrossRef]

12. Radenović, S.; Vetro, F.; Vujaković, J. An alternative and easy approach to fixed point results via simulation functions. Demonstratio Math. 2017, 50, 224-231. [CrossRef]

13. Radenović, S.; Kadelburg, Z.; Jandrlić, D.; Jandrlić, A. Some results on weakly contractive maps. Bull. Iranian Math. Soc. 2012, 38, 625-645.

14. Abbas, M.; Jungck, J. Common fixed point results for non-commuting mappings without continuity in cone metric spaces. J. Math. Anal. Appl. 2008, 341, 416-420. [CrossRef] 
15. Jungck, G. Commuting maps and fixed points. Amer. Math. Monthly 1976, 83, 261-263. [CrossRef]

16. Olgun, M.; Bicer, O.; Alyildiz, T. A new aspect to Picard operators with simulation functions. Turk. J. Math. 2016, 40, 832-837. [CrossRef] 